\title{
Research on active response proactive elderly care service model based on user interest atlas discovery and personalized recommendation
}

\author{
Mengfang Zha ${ }^{1}$, Yibing Song ${ }^{1}$, Qiang Tai ${ }^{1}$, Ying Yao ${ }^{2}$ \\ ${ }^{1}$ Beijing Branch of State Grid Information and Communication Industry Group Co. LTD, Beijing, China \\ ${ }^{2}$ Zhejiang Electric Power Research Institute of State Grid Co., LTD, Beijing, China
}

Keywords: Internet+, proactive elderly care service, interest graph, personalized recommendation, cloud computing.

\begin{abstract}
The government's "lower-level groups pay more attention to the public's needs and pay less attention to", the free and scattered social pension resources, and the relaxation of the atmosphere of the proactive elderly care service market. Service requirements. As one of the "Internet + old-age service" methods, old-age service. 2. To a certain extent, it can play the role of the optimization and integration of the Internet in the resource allocation of proactive elderly care services. On the basis of combing the theoretical discussion and practice of elder care service supply and the application of the Internet in the elder care service in detail, the elder care service is provided. 20 The key elements such as content, functions, framework and guarantee of the operation platform were discussed, with a view to driving the provision of offline services through the operation and consumption of online old-age care services, and shaping orderly competition while meeting the needs of old-age care The environment to guide the rudiment of the marketization of old-age services, and ultimately realize the true socialization and marketization of old-age services. Drawing on the theories of Web 2.0, social networks, complex networks, ontology, and cloud computing, a personalized recommendation system structure based on user interest graphs is designed, the principle of recommendation based on user interest graphs is clarified, and user interest graph generation is proposed The integration method and the dynamic evolution and feedback mechanism of user interest graphs have improved the recommendation quality and accuracy of the recommendation system.
\end{abstract}

\section{Introduction}

Old-age care services stem from the needs of the elderly and finally the supply of services. This supply can be said to be an important component of China's supply side, especially when it is faced with a huge and predictable demand for old-age services [1]. A properly constructed and well-functioning old-age service supply system is even more important. The supply side is a key link for the realization of old-age services. The participation of supply entities, the allocation of supply resources and the setting of supply methods affect the supply quantity and quality of old-age services and ultimately affect the effective satisfaction of old-age needs and the development and stability of the elderly people's later life and even economic society[2].

However, under the trend of a sharp increase in the demand for old-age service, the government's guiding role is still insufficient, the degree of marketization is still not high, and social participation is still limited, so that the practice of old-age service is still generally government-driven[3].

And the government's "lower-level groups pay attention to more people, less attention to needs, free and scattered social pension resources, and a loose atmosphere in the proactive elderly care service market. Issues such as the mismatch between the supply and demand of proactive elderly care services and insufficient market vitality make it difficult to effectively meet the "socialization" of most "home" elderly Demand for old-age services [4]. Especially as China's aging continues to intensify, market-oriented, fast, convenient and effective matching of the supply and demand of old-age services that requires social forces to respond to the growing demand for old-age services. The "Internet+" has just become an old-age service [5]. The face-to-face connection creates an 
orderly competitive environment and guides the marketization of proactive elderly care services. The formation of the prototype [6].

Most of the existing recommendation algorithm research is based on customer characteristics or purchase records. The recommendation algorithm still has problems such as cold start, sparseness, accuracy and diversity. With the development of Web2.0, social networks, big data and cloud computing technologies, online shopping users can publish valuable comment information through more channels (including online shopping sites, blogs, forums, etc.), as expressed in these comments Your opinions and emotions will become an important reference index for potential customers when making shopping choices. In addition, the various behavior information of users in the online shopping environment will also reflect their own personalized information, and correct analysis and effective response to them are of vital importance to the development of online shopping websites [7].The recommendation theory based on the interest graph brings hope to the breakthrough of the qualitative recommendation system. It is based on the subscriptions (such as on Twitter), the purchased goods (such as on Amazon), the ratings (such as on the video website Netflix), Running searches (such as on Google) or comments of certain flavors (such as on Huneh) integrate to generate interest graphs, and efficiently recommend target users based on the similarity of user interests, which can largely solve the problems of other user models Data sparsity and cold start issues can provide diversity recommendations. Therefore, it is necessary to introduce the concept of user interest to make recommendations.

\section{Proactive elderly care service and interest map}

\subsection{Research on the provision of proactive elderly care services}

Most of the domestic and foreign research on elderly care services stems from the response to the problem of elderly group care brought about by the aging population, aging population and weakened family functions [8]. Throughout the existing research, there are aspects such as the old-age service system (model, system), old-age service demand (subject, content, evaluation and forecast), old-age service supply (subject, resources and methods).

These documents all reflect the proactive elderly care service The supply pattern of "the government supports the basic civilian power as the main body" (Dang Junwu 2013) provides policy guidance and operational space for inspiring social forces to participate in the provision of proactive elderly care services [9].

\subsection{Interest graph}

Recommendations based on interest graphs have thus become a research hotspot in recent years. Unlike social graphs, which show relationships between people, interest graphs care about things, not people. In addition, social graphs are basically static (except for occasionally making friends and deleting friends), while interest graphs are flexible and dynamic for individual users. The Irish National Galway University Digital Enterprise Research Center (DERI) has proposed a cross-site semantic-based modeling of user interest graphs. They integrate user information shared on private websites to obtain users' complete interest graphs and use hybrid link prediction. And content-based diffusion activation methods are recommended. BERKOVSKY and others proposed a general user interest model integrated arbitration framework, using data obtained from other recommendation systems to build a fused user model, even if some source data is missing, it can still achieve better recommendation results. The corporate world has begun to try interest graphs. Gravity has developed multiple interest websites and services for multiple websites at the same time. It can track the behavior of users on all its service websites, and form an interest through the analysis of reading history and interests. Combining interest graph theory, ontology theory, cloud computing technology and e-commerce recommendation technology, the author has designed an interest graph based recommendation system (IGRS) based on interest graph, which can satisfy the effective acquisition and aggregation of commodities in a massive data environment And intelligent recommendation requirements, and improve the scale, quality and real-time nature of e-commerce recommendation 
systems.

\section{Platform design}

\subsection{Setting of platform functions}

Taking into account the above-mentioned particularities, there should be a certain tendency in designing the form and function of the proactive elderly care service operation platform. All in all, the proactive elderly care service operation platform should realize the information release, automatic matching, service agreement, multi-party feedback, and integration of supply and demand big data between the service supply and demand sides (see Figure 1).

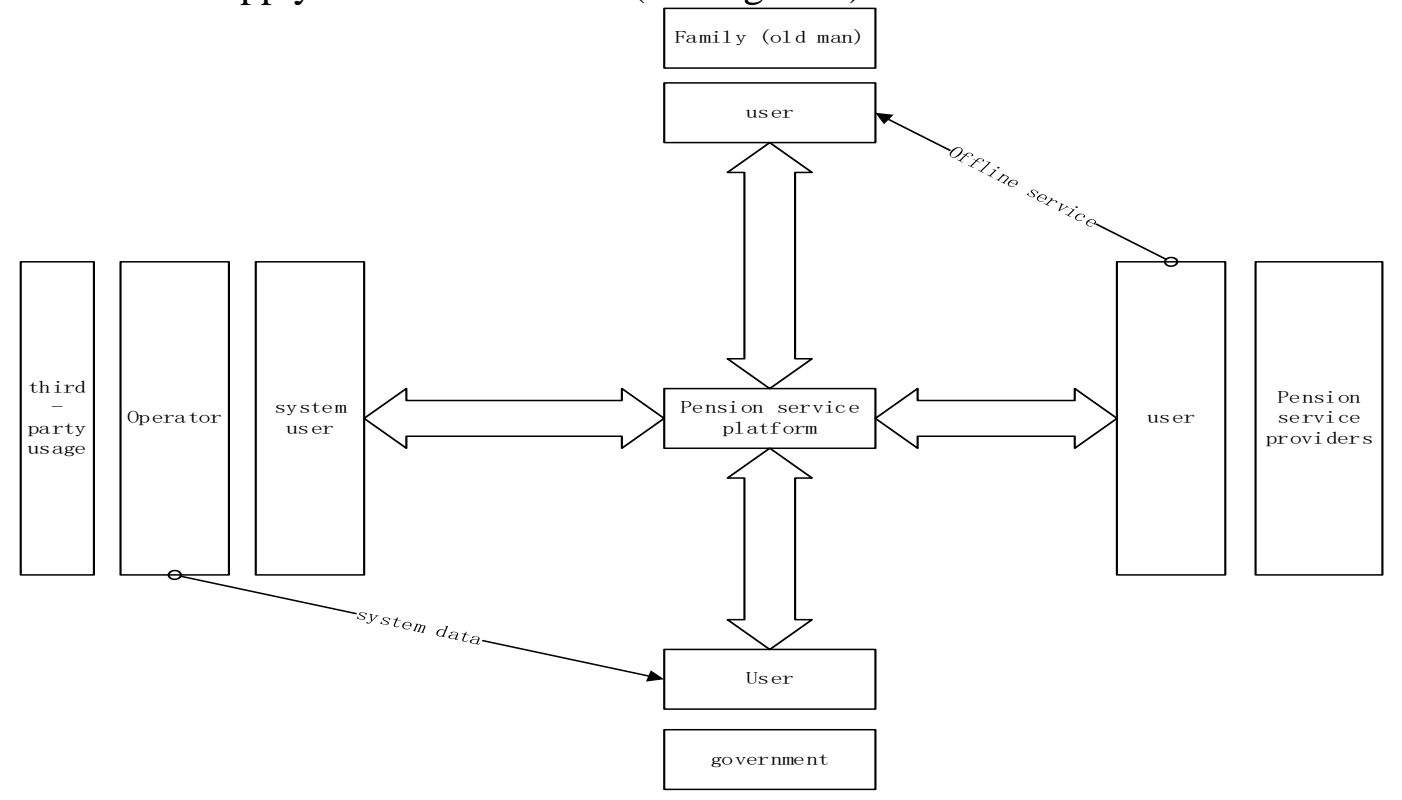

Figure 1 . The function setting of the proactive elderly care service 020 platform
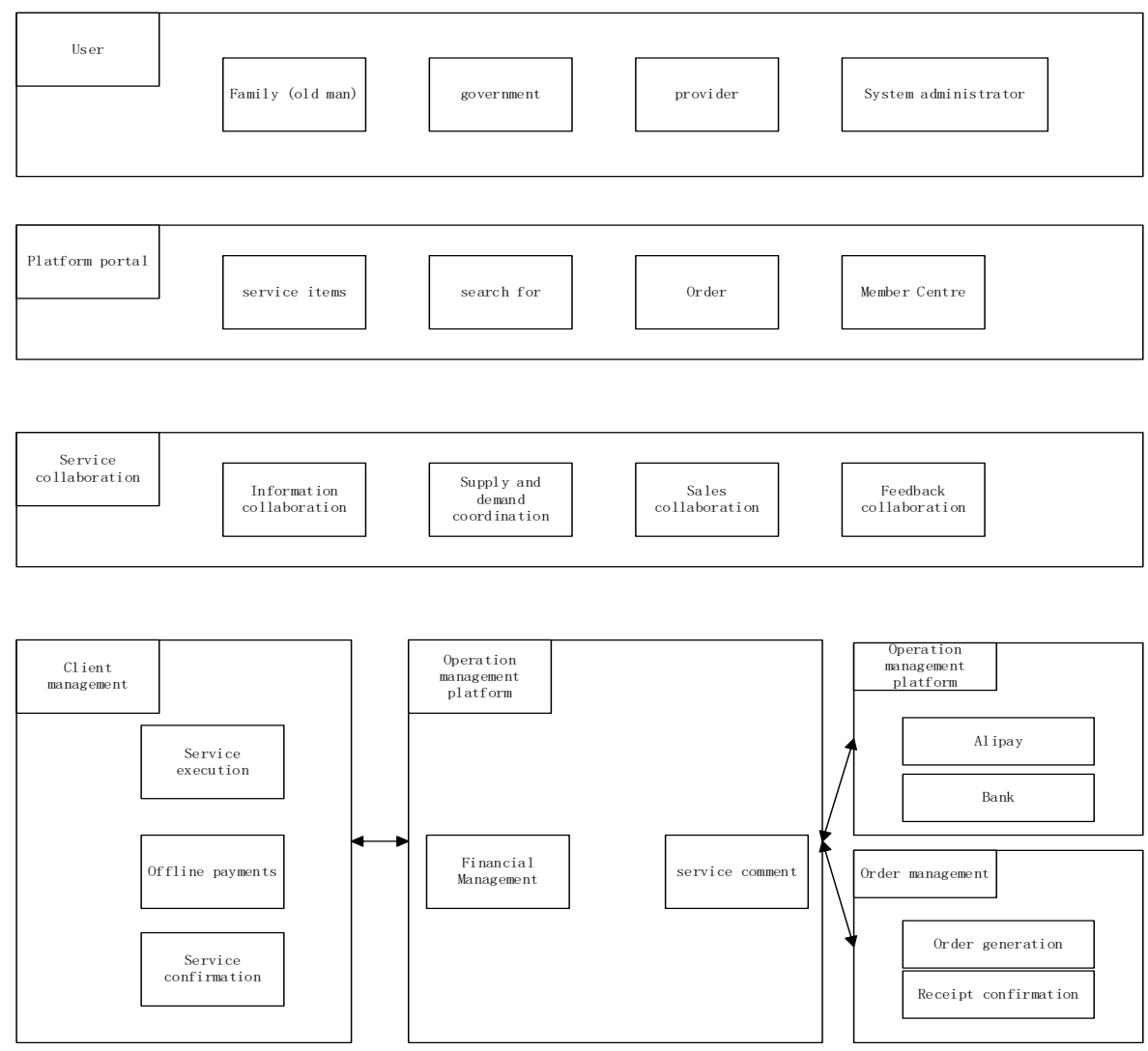

Figure 2. 020 platform architecture of proactive elderly care service 


\subsection{Construction of platform framework}

Drawing on the general requirements of the framework of the 020 e-commerce platform and integrating the special requirements of the proactive elderly care service 020 operating platform, on the basis of ensuring the realization of various functions of the service platform, the network architecture is used to build the overall architecture of the platform and the design of function points, including the design system workflow and database ( Terminal and server), data communication model, user interface and interaction design and repeated revisions to ensure the scientificity and completeness of the platform modules, overall architecture, operation process, and specific design (see Figure 2 for the proactive elderly care service 020 platform architecture).

\subsection{System principle}

Based on the user's interest graph, IGRS first recommends the concept of commodities, and then recommends specific examples of commodities according to the user's preferences, so as to achieve highly efficient and accurate recommendations. By constructing a three-part graph of user-interest-commodity concepts with weights, computing user semantic similarity to generate user candidate interest sets, and then using Bayesian classification algorithm to recommend product concept sets to target users based on the two-dimensional matrix of interest-commodity concepts.

\subsection{Structure design of personalized recommendation system}

The user's interest is not static. For its evolution process, the technical principle of dynamic evolution of user interest graph and feedback mechanism is proposed. First, construct a user-interest bipartite graph based on the user interest graph, and refer to methods such as complex network graph structure link prediction to recommend or predict new interests to users.

(1) External interface layer. The external interface layer can realize the interaction between the personalized recommendation server based on the user's interest graph and the user; provide the interest graph ontology database and the management interface of each database; and provide the interface with the cloud resource scheduling subsystem.

(2) Personalized recommendation subsystem based on user interest graph. The subsystem provides user interest graph analyzer, similarity and weight calculator, three-part graph builder, user preference miner, situational awareness receiver, personalized recommendation engine and 3 recommendation modules.

(3) User interest graph generation subsystem. This subsystem is responsible for user's entire network data acquisition, user interest extraction, local interest graph generation, global interest graph integration, and interest graph optimization. The system develops corresponding adapters based on API interfaces provided by sites such as social networking sites, social tagging sites, shopping sites, and associated data cloud LDBs, obtains relevant data sets, and extracts data that can represent user interests (such as basic information, products, hobbies, Labels, attributes, situational awareness, etc.).

\section{Summary}

The "Internet + pension" model came into being in the context of "Internet +". Its immeasurable development potential and its predictable profit space will definitely attract a large number of social forces into the elderly care service industry. The market-oriented way to develop the old-age service industry has been opened, and the reality requires that the previous government departments change the management of old-age work to avoid policy fragmentation caused by the cross-cutting policies between the civil affairs department, the commercial department, the Development and Reform Commission, and the Aging Committee. Problems, the development of the "Internet + old-age care" model requires unified policy guidance. In the face of new issues in the development of the old-age service industry, with the authenticity and timeliness of the policy to reverse the plight of one-to-one in the field of old-age care, the use of policies Regulation promotes the scientific and standardized development of the proactive elderly care service industry. 


\section{Acknowledgement}

Science and Technology Project of State Grid Headquarters: R\&d and application demonstration of key technologies of highly trusted Intelligent perception interactive integrated Service System (No. : 5211DS18002J)

\section{References}

[1] Song X . Personalized Recommendation Driven by Information Flow[J]. Proc of the Acm Sigir, 2006, 27(6):509-516.

[2] Xiaodan Song, Belle L. Tseng, Ching-Yung Lin, et al. Personalized recommendation driven by information flow.[C]// Sigir: International Acm Sigir Conference on Research \& Development in Information Retrieval. DBLP, 2006.

[3] Patel J, Strickman M . Statistical personalized recommendation system: US 2006.

[4] Ardissono, Liliana, Goy, Anna, Petrone, Giovanna, et al. Intrigue: Personalized recommendation of tourist attractions for desktop and hand held devices [J]. Applied Artificial Intelligence, 2003, 17(8-9):687-714.

[5] Gan M , Jiang R . Improving accuracy and diversity of personalized recommendation through power law adjustments of user similarities[J]. Decision support systems, 2013, 55(3):811-821.

[6] Zhang Z K , Zhou T , Zhang Y C . Personalized Recommendation via Integrated Diffusion on User-Item-Tag Tripartite Graphs[J]. Physica A: Statistical Mechanics and its Applications, 2010, 389( 1):179-186.

[7] Kim H K , Kim J K , Ryu Y U . Personalized Recommendation over a Customer Network for Ubiquitous Shopping[J]. IEEE Transactions on Services Computing, 2009, 2(2):140-151.

[8] Kim H K, Kim J K, Ryu Y U. Personalized Recommendation over a Customer Network for Ubiquitous Shopping[J]. IEEE Transactions on Services Computing, 2009, 2(2):140-151.

[9] Da-Song D, Kai W. A comparative study of the home-based proactive elderly care services [J]. Journal of hebei normal university (philosophy and social sciences edition), 2015.

[10] \&NA. CONSULTANT SERVICE FOR THE AGED[J]. Journal of Nervous \& Mental Disease, 1956, 124(2):216. 